\title{
NECESSARY CONDITIONS FOR OPTIMAL CONTROL OF ELLIPTIC SYSTEMS
}

\author{
HANG GAO ${ }^{1}$ and XUNJING $\mathrm{LI}^{2}$
}

(Received 29 March 1996; revised 27 January 1998)

\begin{abstract}
In this paper, we consider the system governed via the coefficients of a semilinear elliptic equation and give the necessary conditions for optimal control. Furthermore, we obtain the necessary conditions for an optimal domain in a domain optimization problem.
\end{abstract}

\section{Formulation of the problem}

In this paper, we consider the system governed via the divergence component of a semilinear elliptic equation. Standard results of optimal control problems for systems governed by elliptic equations with distributed control can be found in $[2,3,5,10,13$, $15,16]$. Casas considers the system governed via the coefficients of a linear elliptic equation in [4] and gives the necessary conditions of optimal control by using convex analysis under the supposition that the phase spaces of both the control and the cost functional are convex. In this paper, the phase space of both the control and the cost functional may not be convex. We will give the necessary conditions of optimal control by using the convexification method and Ekeland's variational principle.

We first consider the following problem: there are two kinds of materials $\mathscr{A}$ and $\mathscr{B}$ (for example, material $\mathscr{A}$ may be oil and material $\mathscr{B}$ may be water). Let the temperatures of $\mathscr{A}$ and $\mathscr{B}$ be given by $y_{\mathscr{A}}$ and $y_{\mathscr{B}}$ respectively. The quantities $\Omega_{\mathscr{A}}$ and $\Omega_{\mathscr{B}}$ represent the domains occupied by $\mathscr{A}$ and $\mathscr{B}$. Assume $\Gamma_{\mathscr{A}}$ (the boundary of $\Omega_{\mathscr{A}}$ ) and $\Gamma_{\mathscr{A}}$ ((the boundary of $\left.\Omega_{\mathscr{B}}\right) \backslash \Gamma_{\mathscr{A}}$ ) are smooth (see Figure 1). Then $y_{\mathscr{A}}$ and

\footnotetext{
${ }^{1}$ Department of Mathematics, Northeast Normal University, Changchun 130024, P. R. China.

${ }^{2}$ Department of Mathematics, Fudan University, Shanghai 200433, P. R. China.

(C) Australian Mathematical Society 2000, Serial-fee code 0334-2700/00
} 


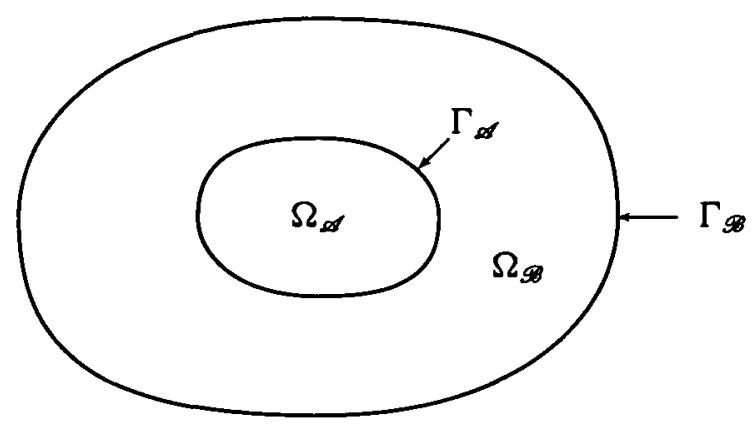

FIGURE 1.

$y_{\mathscr{B}}$ satisfy the equations

$$
\begin{cases}-a \Delta y_{\mathscr{A}}(x)=f(x), & x \in \Omega_{\mathscr{A}}, \\ -b \Delta y_{\mathscr{B}}(x)=f(x), & x \in \Omega_{\mathscr{B}}, \\ \left.y_{\mathscr{B}}\right|_{\Gamma_{\mathscr{O}}}=0, & \\ \left.a \frac{\partial}{\partial n} y_{\mathscr{A}}\right|_{\Gamma_{\mathscr{A}}}=\left.b \frac{\partial}{\partial n} y_{\mathscr{D}}\right|_{\Gamma_{\mathscr{A}}}, & \left.y_{\mathscr{A}}\right|_{\Gamma_{\mathscr{A}}}=\left.y_{\mathscr{B}}\right|_{\Gamma_{\mathscr{A}}},\end{cases}
$$

where $b>a>0$. We know that there exists a unique classical solution $y_{\mathscr{A}}$ and $y_{\mathscr{B}}$ for Problem (1.1) if $f(\cdot) \in C^{0, \alpha}(G)$ for $0<\alpha<1$. We now give a cost functional

$$
F\left(\Omega_{\mathscr{A}}\right)=\int_{\Omega_{\mathscr{A}}} f^{0}\left(x, y_{\mathscr{A}}(x), \nabla y_{\mathscr{A}}(x)\right) d x+\int_{\Omega_{\mathscr{S}}} f^{0}\left(x, y_{\mathscr{B}}(x), \nabla y_{\mathscr{B}}(x)\right) d x
$$

and a set

$$
\Pi=\left\{\Omega_{\star}\left|\Omega_{\star} \subset G,\right| \Omega_{\infty} \mid=1, \Gamma_{\infty} \text { is smooth }\right\},
$$

where $|E|=$ meas $E, G$ is a fixed domain and $G=\Omega_{\mathscr{A}} \cup \Omega_{\mathscr{B}}$.

We can raise the following domain optimization problem.

PROBLEM D. Find a domain $\tilde{\Omega}_{\propto} \in \Pi$, such that

$$
F\left(\tilde{\Omega}_{\infty}\right)=\inf \left\{F\left(\Omega_{\infty}\right) \mid \Omega_{\infty} \in \Pi\right\} .
$$

If there exists a domain $\tilde{\Omega}_{\star}$ such that (1.4) holds, we say that the domain $\tilde{\Omega}_{\infty}$ is an optimal domain.

We now introduce a function

$$
u(x)=a \chi_{\Omega_{\alpha}}(x)+b \chi_{\Omega_{g t}}(x) .
$$


Problem (1.1) can then be written as

$$
\left\{\begin{array}{l}
-\operatorname{div}\left(u(x) \nabla y_{u}(x)\right)=f(x), \quad x \in G, \\
y_{u} \mid \Gamma_{G}=0
\end{array}\right.
$$

that is,

$$
y_{u}(x)= \begin{cases}y_{\mathscr{A}}(x), & x \in \Omega_{\mathscr{A}}, \\ y_{\mathscr{B}}(x), & x \in \Omega_{\mathscr{B}} .\end{cases}
$$

The cost functional (1.2) becomes

$$
J(u)=\int_{G} f^{0}\left(x, y_{u}(x), \nabla y_{u}(x)\right) d x .
$$

Let

$$
\mathscr{W}=\left\{u(x)=a \chi_{\Omega_{\mathscr{d}}}(x)+b \chi_{\Omega_{\mathscr{g}}}(x) \mid \Omega_{\mathscr{A}} \in \Pi\right\} .
$$

We can now raise the optimal control problem corresponding to Problem D.

Problem C. Find a control $\bar{u} \in \mathscr{W}$, such that

$$
J(\bar{u})=\inf \{J(u) \mid u \in \mathscr{W}\} .
$$

In this practical problem, the control variable is involved in the coefficient, the admissible control set $\mathscr{W}$ is not convex and the cost functional (1.8) may not be convex.

In this paper, we will discuss a more general system:

$$
\left\{\begin{array}{l}
-\sum_{i, j=1}^{n} \frac{\partial}{\partial x_{i}}\left(a_{i j}(x, u(x)) \frac{\partial}{\partial x_{j}} y(x)\right)=f(x, y(x), u(x)), \\
\left.y\right|_{\Gamma_{\Omega}}=0,
\end{array}\right.
$$

where $\Omega\left(\subset R^{n}\right)$ is a bounded domain with smooth boundary $\Gamma_{\Omega}, u(x) \in U$ is a control function, $U \subset R^{m}$ is a bounded closed set and co $U$ stands for the convex hull of the $U$.

We denote the set of all admissible controls by $\mathscr{U}_{\text {ad }}$, that is,

$$
\mathscr{U}_{\text {ad }}=\{u(x) \in U \mid u(\cdot) \text { is measurable on } \Omega\} .
$$

If for any $u(\cdot) \in \mathscr{U}_{\mathrm{ad}}, y(x)=y(x ; u)$ is a solution of Problem (1.11), we can define the cost functional

$$
J(u)=\int_{\Omega} f^{0}(x, y(x ; u), \nabla y(x ; u), u(x)) d x .
$$

Our optimal control problem can be stated as follows. 
PROBlem E. Find a $\bar{u}(\cdot) \in \mathscr{U}_{\text {ad }}$, such that

$$
J(\bar{u}(\cdot))=\inf \left\{J(u(\cdot)) \mid u(\cdot) \in \mathscr{U}_{\mathrm{ad}}\right\},
$$

where $J(u(\cdot))$ is given by (1.13).

Any admissible control $\bar{u}(\cdot)$ satisfying (1.14) is called an optimal control for Problem $\mathrm{E}$; the corresponding state $\bar{y}(\cdot)$ is called an optimal state and the pair $(\bar{y}(\cdot), \bar{u}(\cdot))$ is referred to as an optimal pair.

\section{Variation of convexification problems}

Let us assume that

(P1) $a_{i j}: \Omega \times \operatorname{co} U \rightarrow R$ satisfies the following conditions.

(1) The quantity $a_{i j}(\cdot, u)$ is bounded measurable on $\Omega$ and $a_{i j}(x, \cdot)$ is Lipschitz continuous of rank $K$ on co $U$.

(2) There exists a constant $\lambda>0$, for any $(x, u) \in \Omega \times \operatorname{co} U$, such that

$$
\sum_{i, j=1}^{n} a_{i j}(x, u) \eta_{i} \eta_{j} \geq \lambda|\eta|^{2}, \quad \forall \eta \in R^{n}
$$

We shall also assume that

(P2) $f: \Omega \times R \times \operatorname{co} U \rightarrow R$ is such that $f(\cdot, y, u)$ is measurable on $\Omega$ and that $f(x, \cdot, \cdot)$ and $f_{y}(x, \cdot, \cdot)$ are Lipschitz continuous of rank $K$ on $R \times$ co $U$. There exists a constant $L>0$, such that

$$
-L \leq f_{y}(x, y, u) \leq 0, \quad \forall(x, y, u) \in \Omega \times R \times \operatorname{co} U,
$$

where $f^{0}(\cdot, y, \zeta, u)$ is measurable on $\Omega$ and $f^{0}(x, \cdot, \cdot, \cdot), f_{y}^{0}(x, \cdot, \cdot, \cdot)$ and $f_{\zeta_{i}}^{0}(x, \cdot, \cdot, \cdot)$ are Lipschitz continuous of rank $K$ on $R \times R^{n} \times$ co $U$.

For any $M>0$, there exists a function $F_{M}(\cdot) \in L^{2}(\Omega)$, such that

$$
\begin{gathered}
|f(x, y, u)|+\left|f_{y}(x, y, u)\right|+\left|f^{0}(x, y, \zeta, u)\right|+\left|f_{y}^{0}(x, y, \zeta, u)\right| \leq\left|F_{M}(x)\right|, \\
|y| \leq M, \quad \forall u \in \operatorname{co} U, \quad \forall \zeta \in R^{n} .
\end{gathered}
$$

In deriving necessary conditions for optimal control, one needs to make certain perturbations for the control and the corresponding variations of the state and the cost functional need to be determined.

We first introduce a new control set

$$
\mathscr{U}=\{u(x) \mid u(\cdot): \Omega \rightarrow \text { co } U \text { is measurable }\} .
$$


DEFINITION 2.1. A function $y(\cdot)$ is called a generalized solution of Problem (1.11) if $y(\cdot) \in H_{0}^{1}(\Omega)$ and for any $\varphi \in H_{0}^{1}(\Omega)$ the following equality holds:

$$
\int_{\Omega} \sum_{i, j=1}^{n} a_{i j}(x, u(x)) \frac{\partial}{\partial x_{j}} y(x) \frac{\partial}{\partial x_{i}} \varphi(x) d x=\int_{\Omega} f(x, y(x), u(x)) \varphi(x) d x .
$$

We have the following lemmas.

LEMMA 2.1. Let (P1)-(P2) hold. For any $u \in \mathscr{U}$, there exists a unique generalized solution $y(x)=y(x ; u) \in H_{0}^{1}(\Omega)$ for Problem (1.11), and there exists a constant $C$ being independent of $u$, such that

$$
\|y\|_{H^{\prime}(\Omega)} \leq C .
$$

Proof. Step 1. We have

$$
|f(x, y(x), u(x))| \leq c|y(x)|+c_{1}(x) .
$$

In fact,

$$
\begin{aligned}
f(x, y(x), u(x)) & =f(x, y(x), u(x))-f(x, 0, u(x))+f(x, 0, u(x)) \\
& =\int_{\Omega} f_{y}(x, \tau y(x), u(x)) d \tau y(x)+f(x, 0, u(x)) .
\end{aligned}
$$

From this equality and condition (P2), we know that the inequality of Step 1 is true. Step 2. For any $y \in H_{0}^{1}(\Omega)$, we have

$$
\begin{aligned}
\int_{\Omega} \sum_{i, j=1}^{n}\left\{a_{i j}(x, u(x)) \frac{\partial}{\partial x_{j}} y(x) \frac{\partial}{\partial x_{i}} y(x) d x\right. \\
\quad-f(x, y(x), u(x)) y(x)\} d x \geq c_{2}\|y\|_{H^{1}(\Omega)}-c_{3} .
\end{aligned}
$$

In fact, for any $y \in H_{0}^{1}(\Omega)$,

$$
\begin{aligned}
\int_{\Omega} \sum_{i, j=1}^{n} & \left\{a_{i j}(x, u(x)) \frac{\partial}{\partial x_{j}} y(x) \frac{\partial}{\partial x_{i}} y(x) d x-f(x, y(x), u(x)) y(x)\right\} d x \\
= & \int_{\Omega} \sum_{i, j=1}^{n}\left\{a_{i j}(x, u(x)) \frac{\partial}{\partial x_{j}} y(x) \frac{\partial}{\partial x_{i}} y(x) d x\right. \\
& \left.-\int_{0}^{1} f_{y}(x, \tau y(x), u(x)) d \tau y^{2}(x)-f(x, 0, u(x)) y(x)\right\} d x \\
\geq & \lambda\|\nabla y\|_{L^{2}(\Omega)}-\varepsilon\|y\|_{L^{2}(\Omega)}-C(\varepsilon)\|f(\cdot, 0, u)\|_{L^{2}(\Omega)} .
\end{aligned}
$$


Since $\|\nabla y\|_{L^{2}(\Omega)} \geq C\|y\|_{L^{2}(\Omega)}$ for any $y \in H_{0}^{1}(\Omega)$, we can choose $\varepsilon$ small enough so that

$$
\lambda\|\nabla y\|_{L^{2}(\Omega)}-\varepsilon\|y\|_{L^{2}(\Omega)} \geq \frac{1}{2} \lambda\|\nabla y\|_{L^{2}(\Omega)} \geq c_{2}\|y\|_{H_{0}^{1}(\Omega)} .
$$

Therefore Step 2. holds.

Secondly, from condition (P2), it is easy to see that

$$
\int_{\Omega}\left\{f\left(x, y_{1}(x), u(x)\right)-f(x, y(x), u(x)) y(x)\right\}\left\{y_{1}(x)-y_{2}(x)\right\} d x \leq 0 .
$$

According to Theorem 9.1 of Chapter 4 in [9], we know that there exists a function $y \in H_{0}^{1}(\Omega)$ which satisfies (2.2).

We can prove the uniqueness of this solution. In fact, suppose $y_{1}(x)$ and $y_{2}(x)$ are the generalized solutions of Problem (1.11). We then have

$$
\begin{aligned}
\int_{\Omega} \sum_{i, j=1}^{n} a_{i j}(x, u(x)) \frac{\partial}{\partial x_{j}}\left(y_{1}(x)-y_{2}(x)\right) \frac{\partial}{\partial x_{i}} \varphi(x) d x \\
\quad=\int_{\Omega}\left[f\left(x, y_{1}(x), u(x)\right)-f\left(x, y_{2}(x), u(x)\right)\right] \varphi(x) d x .
\end{aligned}
$$

In particular, let $\varphi(x)=y_{1}(x)-y_{2}(x)$. We now have

$$
\begin{aligned}
0 & \leq \lambda\left\|\nabla\left(y_{1}-y_{2}\right)\right\|_{L^{2}(\Omega)} \\
& \leq \int_{\Omega} \sum_{i, j=1}^{n} a_{i j}(x, u(x)) \frac{\partial}{\partial x_{j}}\left(y_{1}(x)-y_{2}(x)\right) \frac{\partial}{\partial x_{i}}\left(y_{1}(x)-y_{2}(x)\right) d x \\
& =\int_{\Omega} \int_{0}^{1} f_{y}\left(x, y_{2}(x)+\tau\left(y_{1}(x)-y_{2}(x)\right), u(x)\right) d \tau\left\{y_{1}(x)-y_{2}(x)\right\}^{2} d x \leq 0 .
\end{aligned}
$$

Therefore $\left\|\nabla\left(y_{1}-y_{2}\right)\right\|_{L^{2}(\Omega)}=0$. That is, $\left\|y_{1}-y_{2}\right\|_{H^{\prime}(\Omega)}=0$, so $y_{1}(x)=y_{2}(x)$.

Using conditions (P1)-(P2), we have

$$
\begin{aligned}
\lambda \int_{\Omega}|\nabla y(x)|^{2} d x & \leq \int_{\Omega} \sum_{i, j=1}^{n} a_{i j}(x, u(x)) \frac{\partial}{\partial x_{j}} y(x) \frac{\partial}{\partial x_{i}} y(x) d x \\
& =\int_{\Omega} f(x, y(x), u(x)) y(x) d x \\
& =\int_{\Omega} f(x, 0, u(x)) y(x) d x+\int_{\Omega} \int_{0}^{1} f_{y}(x, \tau y(x), u(x)) d \tau y^{2}(x) d x \\
& \leq C\|f(\cdot, 0, u(\cdot))\|_{L^{2}(\Omega)}\|y\|_{L^{2}(\Omega)} .
\end{aligned}
$$

From Poincaré's inequality, we have

$$
\lambda\|\nabla y(\cdot)\|_{L^{2}(\Omega)}^{2} \leq C\|F(\cdot)\|_{L^{2}(\Omega)}\|\nabla y(\cdot)\|_{L^{2}(\Omega)},
$$

so the inequality (2.3) holds. 
Let $\bar{u}, v \in \mathscr{U}, \bar{y}(\cdot)=y(\cdot, \bar{u})$ and $y_{v}(\cdot)=y(\cdot, v)$ be the generalized solutions of Problem (1.11) corresponding to $\bar{u}(\cdot)$ and $v(\cdot)$, respectively.

LEMMA 2.2. Let (P1)-(P2) hold. We have the following estimate

$$
\left\|\bar{y}(\cdot)-y_{v}(\cdot)\right\|_{H^{\prime}(\Omega)} \leq C\|\bar{u}-v\|_{L^{\infty}(\Omega)},
$$

where the constant $C$ is independent of $\vec{u}(\cdot)$ and $v(\cdot)$.

PROOF. From (1.11), we have

$$
\begin{aligned}
-\sum_{i, j=1}^{n} & \frac{\partial}{\partial x_{i}}\left(a_{i j}(x, v(x)) \frac{\partial}{\partial x_{j}}\left(y_{v}(x)-\bar{y}(x)\right)\right) \\
= & \sum_{i, j=1}^{n} \frac{\partial}{\partial x_{i}}\left(a_{i j}(x, v(x))-a_{i j}(x, \bar{u}(x))\right) \frac{\partial}{\partial x_{j}} \bar{y}(x) \\
& +\left[f\left(x, y_{v}(x), v(x)\right)-f(x, \bar{y}(x), \bar{u}(x))\right] \\
= & \sum_{i, j=1}^{n} \frac{\partial}{\partial x_{i}}\left(a_{i j}(x, v(x))-a_{i j}(x, \bar{u}(x))\right) \frac{\partial}{\partial x_{j}} \bar{y}(x) \\
& +\int_{0}^{1} f_{y}\left(x, \bar{y}(x)+\tau\left(y_{v}(x)-\bar{y}(x)\right), v(x)\right) d \tau\left(y_{v}(x)-\bar{y}(x)\right) \\
& +[f(x, \bar{y}(x), v(x))-f(x, \bar{y}(x), \bar{u}(x))] .
\end{aligned}
$$

Multiplying (2.5) by $y_{v}(x)-\bar{y}(x)$ and integrating the resulting relation over $\Omega$, we have

$$
\begin{aligned}
& \lambda\left\|\nabla\left(y_{v}-\bar{y}\right)\right\|_{L^{2}(\Omega)}^{2} \\
& \leq \int_{\Omega} \sum_{i, j=1}^{n}\left[a_{i j}(x, \bar{u}(x))-a_{i j}(x, v(x))\right] \frac{\partial}{\partial x_{j}} \bar{y}(x) \frac{\partial}{\partial x_{i}}\left(y_{v}(x)-\bar{y}(x)\right) d x \\
& \quad+\int_{\Omega} \int_{0}^{1} f_{y}\left(x, \bar{y}(x)+\tau\left(y_{v}(x)-\bar{y}(x)\right), v(x)\right) d \tau\left(y_{v}(x)-\bar{y}(x)\right)^{2} d x \\
& \quad+\int_{\Omega}[f(x, \bar{y}(x), v(x))-f(x, \bar{y}(x), \bar{u}(x))]\left(y_{v}(x)-\bar{y}(x)\right) d x \\
& \leq K\|\bar{u}-v\|_{L^{\infty}(\Omega)}\|\nabla \bar{y}\|_{L^{2}(\Omega)}\left\|\nabla\left(y_{v}-\bar{y}\right)\right\|_{L^{2}(\Omega)} \\
&+K\|\bar{u}-v\|_{L^{\infty}(\Omega)}\left\|y_{v}-\bar{y}\right\|_{L^{2}(\Omega)} \\
& \leq C\|\bar{u}-v\|_{L^{\infty}(\Omega)}\left\|\nabla\left(y_{v}-\bar{y}\right)\right\|_{L^{2}(\Omega)} .
\end{aligned}
$$

We thus obtain the result of Lemma 2.2. 
Let $z(\cdot) \in H_{0}^{1}(\Omega)$ satisfy the following equation

$$
\left\{\begin{aligned}
-\sum_{i, j=1}^{n} & \frac{\partial}{\partial x_{i}}\left(a_{i j}(x, \bar{u}(x)) \frac{\partial}{\partial x_{j}} z(x)\right) \\
= & f_{y}(x, \bar{y}(x), \bar{u}(x)) z(x) \\
& +\sum_{i, j=1}^{n} \frac{\partial}{\partial x_{i}}\left(a_{i j}(x, v(x))-a_{i j}(x, \bar{u}(x))\right) \frac{\partial}{\partial x_{j}} \bar{y}(x) \\
& +[f(x, \bar{y}(x), v(x))-f(x, \bar{y}(x), \bar{u}(x))], \\
z \mid \Gamma_{\mathrm{s}}= & 0
\end{aligned}\right.
$$

and let

$$
\begin{aligned}
z^{0}= & \int_{\Omega}\left\{f_{y}^{0}(x, \bar{y}(x), \nabla \bar{y}(x), \bar{u}(x)) z(x)+\sum_{i=1}^{n} f_{\zeta_{i}}^{0}(x, \bar{y}(x), \nabla \bar{y}(x), \bar{u}(x)) \frac{\partial}{\partial x_{i}} z(x)\right. \\
& \left.+f^{0}(x, \bar{y}(x), \nabla \bar{y}(x), v(x))-f^{0}(x, \bar{y}(x), \nabla \bar{y}(x), \bar{u}(x))\right\} d x .
\end{aligned}
$$

REMARK 2.1. We see that the solutions $z(\cdot)$ of $(2.6)$ and $z^{0}$ defined by (2.7) are dependent on the choice of $v, \bar{u} \in \mathscr{U}$. If $\bar{u}$ is fixed, then we can denote $z(\cdot)=z(\cdot, v)$ and $z^{0}=z^{0}(v)$. Multiplying (2.6) by $z(x)$ and integrating the resulting relation over $\Omega$, we have

$$
\begin{aligned}
\lambda\|\nabla z\|_{L^{2}(\Omega)}^{2} & \leq K\|\bar{u}-v\|_{L^{\infty}(\Omega)}\|\nabla \bar{y}\|_{L^{2}(\Omega)}\|\nabla z\|_{L^{2}(\Omega)}+K\|\bar{u}-v\|_{L^{\infty}(\Omega)}\|z\|_{L^{2}(\Omega)} \\
& \leq C\|\bar{u}-v\|_{L^{\infty}(\Omega)}\|\nabla z\|_{L^{2}(\Omega)} .
\end{aligned}
$$

Thus, we have

$$
\|z\|_{H^{1}(\Omega)} \leq C\|\bar{u}-v\|_{L^{\infty}(\Omega)} .
$$

THEOREM 2.1. Suppose that conditions (P1)-(P2) hold. Then

$$
\begin{aligned}
y_{v}(x) & =\bar{y}(x)+z(x)+r(x), \\
J(v) & =J(\bar{u})+z^{0}+r^{0}
\end{aligned}
$$

and

$$
\|r\|_{H^{1}(\Omega)}=o\left(\|v-\bar{u}\|_{L^{\infty}(\Omega)}\right), \quad\left|r^{0}\right|=o\left(\|v-\bar{u}\|_{L^{\infty}(\Omega)}\right) .
$$


PROOF. From (2.5) and (2.6), we obtain

$$
\left\{\begin{array}{l}
-\sum_{i, j=1}^{n} \frac{\partial}{\partial x_{i}}\left(a_{i j}(x, v(x)) \frac{\partial}{\partial x_{j}}\left(y_{v}(x)-\bar{y}(x)-z(x)\right)\right) \\
=\sum_{i, j=1}^{n}\left[\frac{\partial}{\partial x_{i}}\left(a_{i j}(x, v(x))-a_{i j}(x, \bar{u}(x))\right) \frac{\partial}{\partial x_{j}} z(x)\right] \\
\quad+\int_{0}^{1} f_{y}\left(x, \bar{y}(x)+\tau\left(y_{v}(x)-\bar{y}(x)\right), v(x)\right) d \tau\left(y_{v}(x)-\bar{y}(x)-z(x)\right) \\
\quad+\int_{0}^{1}\left[f_{y}\left(x, \bar{y}(x)+\tau\left(y_{v}(x)-\bar{y}(x)\right), v(x)\right)-f_{y}(x, \bar{y}(x), \bar{u}(x))\right] d \tau z(x), \\
\left.\left(y_{v}-\bar{y}-z\right)\right|_{\Gamma_{\Omega}}=0 .
\end{array}\right.
$$

Multiplying (2.12) by $y_{v}(x)-\bar{y}(x)-z(x)$ and integrating the resulting relation over $\Omega$, we have

$$
\begin{aligned}
& \lambda\left\|\nabla\left(y_{v}-\bar{y}-z\right)\right\|_{L^{2}(\Omega)}^{2} \\
& \leq \int_{\Omega} \sum_{i, j=1}^{n}\left[a_{i j}(x, \bar{u}(x))-a_{i j}(x, v(x))\right] \frac{\partial}{\partial x_{j}} z(x) \frac{\partial}{\partial x_{i}}\left(y_{v}(x)-\bar{y}(x)-z(x)\right) d x \\
& \quad+\int_{\Omega} \int_{0}^{1} f_{y}\left(x, \bar{y}(x)+\tau\left(y_{v}(x)-\bar{y}(x)\right), v(x)\right) d \tau\left(y_{v}(x)-\bar{y}(x)-z(x)\right)^{2} d x \\
& \quad+\int_{\Omega} \int_{0}^{1}\left[f_{y}\left(x, \bar{y}(x)+\tau\left(y_{v}(x)-\bar{y}(x)\right), v(x)\right)-f_{y}(x, \bar{y}(x), \bar{u}(x))\right] d \tau \\
& \quad \times z(x)\left(y_{v}(x)-\bar{y}(x)-z(x)\right) d x \\
& \leq K\|\bar{u}-v\|_{L^{\infty}(\Omega)}\|\nabla z\|_{L^{2}(\Omega)}\left\|\nabla\left(y_{v}-\bar{y}-z\right)\right\|_{L^{2}(\Omega)} \\
&+K\|\bar{u}-v\|_{L^{\infty}(\Omega)}\|z\|_{L^{2}(\Omega)}\left\|y_{v}-\bar{y}-z\right\|_{L^{2}(\Omega)} \\
& \leq C\|\bar{u}-v\|_{L^{\infty}(\Omega)}^{2}\left\|\nabla\left(y_{v}-\bar{y}-z\right)\right\|_{L^{2}(\Omega) .}
\end{aligned}
$$

From this inequality, we obtain

$$
\|r\|_{H^{1}(\Omega)}=\left\|y_{v}-\bar{y}-z\right\|_{H^{\prime}(\Omega)}=o\left(\|v-\bar{u}\|_{L^{\infty}(\Omega)}\right) .
$$

Furthermore, we may calculate directly

$$
\begin{aligned}
J(v)- & J(\bar{u}) \\
= & \int_{\Omega}\left[f^{0}\left(x, y_{v}(x), \nabla y_{v}(x), v(x)\right)-f^{0}(x, \bar{y}(x), \nabla \bar{y}(x), \bar{u}(x))\right] d x \\
= & \int_{\Omega}\left[f^{0}\left(x, y_{v}(x), \nabla y_{v}(x), v(x)\right)-f^{0}\left(x, \bar{y}(x), \nabla y_{v}(x), v(x)\right)\right] d x \\
& +\int_{\Omega}\left[f^{0}\left(x, \bar{y}(x), \nabla y_{v}(x), v(x)\right)-f^{0}(x, \bar{y}(x), \nabla \bar{y}(x), v(x))\right] d x
\end{aligned}
$$




$$
\begin{aligned}
& +\int_{\Omega}\left[f^{0}(x, \bar{y}(x), \nabla \bar{y}(x), v(x))-f^{0}(x, \bar{y}(x), \nabla \bar{y}(x), \bar{u}(x))\right] d x \\
& =\int_{\Omega} \int_{0}^{1} f_{y}^{0}\left(x, \bar{y}(x)+\tau\left(y_{v}(x)-\bar{y}(x)\right), \nabla y_{v}(x), v(x)\right) d \tau\left(y_{v}(x)-\bar{y}(x)\right) d x \\
& +\int_{\Omega} \sum_{i=1}^{n} \int_{0}^{1} f_{\zeta_{i}}^{0}\left(x, \bar{y}(x), \frac{\partial}{\partial x_{1}} \bar{y}(x), \ldots, \frac{\partial}{\partial x_{i}} \bar{y}(x)\right. \\
& \left.+\tau \frac{\partial}{\partial x_{i}}\left(y_{v}(x)-\bar{y}(x)\right), \ldots, \frac{\partial}{\partial x_{n}} y_{v}(x), v(x)\right) d \tau \frac{\partial}{\partial x_{i}}\left(y_{v}(x)-\bar{y}(x)\right) d x \\
& +\int_{\Omega}\left[f^{0}(x, \bar{y}(x), \nabla \bar{y}(x), v(x))-f^{0}(x, \bar{y}(x), \nabla \bar{y}(x), \bar{u}(x))\right] d x .
\end{aligned}
$$

From (2.9), we have

$$
\begin{aligned}
J(v) & -J(\bar{u}) \\
= & \int_{\Omega} \int_{0}^{1} f_{y}^{0}\left(x, \bar{y}(x)+\tau\left(y_{v}(x)-\bar{y}(x)\right), \nabla y_{v}(x), v(x)\right) d \tau z(x) d x \\
& +\int_{\Omega} \sum_{i=1}^{n} \int_{0}^{l} f_{\zeta_{i}}^{0}\left(x, \bar{y}(x), \frac{\partial}{\partial x_{1}} \bar{y}(x), \ldots, \frac{\partial}{\partial x_{i}} \bar{y}(x)\right. \\
& \left.+\tau \frac{\partial}{\partial x_{i}}\left(y_{v}(x)-\bar{y}(x)\right), \ldots, \frac{\partial}{\partial x_{n}} y_{v}(x), v(x)\right) d \tau \frac{\partial}{\partial x_{i}} z(x) d x \\
& +\int_{\Omega}\left[f^{0}(x, \bar{y}(x), \nabla \bar{y}(x), v(x))-f^{0}(x, \bar{y}(x), \nabla \bar{y}(x), \bar{u}(x))\right] d x+o\left(\|v-\bar{u}\|_{L^{\infty}(\Omega)}\right) \\
= & z^{0}+\int_{\Omega} \int_{0}^{1}\left[f_{y}^{0}\left(x, \bar{y}(x)+\tau\left(y_{v}(x)-\bar{y}(x)\right), \nabla y_{v}(x), v(x)\right)\right. \\
& \left.-f_{y}^{0}(x, \bar{y}(x), \nabla \bar{y}(x), \bar{u}(x))\right] d \tau z(x) d x \\
& +\int_{\Omega} \sum_{i=1}^{n} \int_{0}^{1}\left[f _ { \zeta _ { i } } ^ { 0 } \left(x, \bar{y}(x), \frac{\partial}{\partial x_{1}} \bar{y}(x), \ldots, \frac{\partial}{\partial x_{i}} \bar{y}(x)\right.\right. \\
& \left.\left.+\tau \frac{\partial}{\partial x_{i}}\left(y_{v}(x)-\bar{y}(x)\right), \ldots, \frac{\partial}{\partial x_{n}} y_{v}(x), v(x)\right)-f_{\zeta_{i}}^{0}(x, \bar{y}(x), \nabla \bar{y}(x), \bar{u}(x))\right] d \tau \\
& \times \frac{\partial}{\partial x_{i}} z(x) d x+o\left(\|v-\bar{u}\|_{L^{\infty}(\Omega)}\right)=z^{0}+o\left(\|v-\bar{u}\|_{L^{\infty}(\Omega)}\right) .
\end{aligned}
$$

Thus Theorem 2.1 is proved.

\section{The case of $U$ being the endpoints set of a cuboid}

In this section, we suppose that $U$ is a set of the end points of a cuboid and discuss the necessary conditions for the optimal control problem with a state constraint. 
Now, suppose $Q \subset H_{0}^{1}(\Omega)$. For any $u(\cdot) \in \mathscr{U}_{\text {ad }}$, there exists a unique function $y_{u}(x)=y(x ; u) \in H_{0}^{1}(\Omega)$, which is the generalized solution of (1.11). We can therefore talk about the state constraint

$$
y_{u}(\cdot) \in Q
$$

We let

$$
\mathscr{U}_{\text {ad }}^{Q}=\left\{u \in \mathscr{U}_{\text {ad }} \mid y(\cdot, u) \in Q\right\} .
$$

Our optimal control problem can be stated as follows.

Problem $E_{Q}$. Find a $\bar{u}(\cdot) \in \mathscr{U}_{\text {ad }}^{Q}$, such that

$$
J(\bar{u}(\cdot))=\inf \left\{J(u(\cdot)) \mid u(\cdot) \in \mathscr{U}_{\mathrm{ad}}^{Q}\right\},
$$

where $J(u(\cdot))$ is given by $(1.13)$.

Any admissible control $\bar{u}(\cdot)$ satisfying (3.1) is called an optimal control for Problem $\mathrm{E}_{Q}$, the corresponding state $\bar{y}(\cdot)$ is called an optimal state and the pair $(\bar{y}(\cdot), \bar{u}(\cdot))$ is referred to as an optimal pair.

It is clear that Problem $\mathrm{E}$ is a special case of Problem $\mathrm{E}_{Q}$.

We first raise the question: Suppose $\bar{u}(x)$ is an optimal control of Problem $E$ and $u_{d}(x)$ is an optimal control of Problems (1.11), (1.13) and (2.1). Then is it true that $\bar{u}(x)=u_{d}(x) ?$

EXAMPLE 3.1. We consider the system given by

$$
\left\{\begin{array}{l}
-\operatorname{div}(u(x) \nabla y(x))=f(x), \quad x \in \Omega, \\
\left.y_{u}\right|_{\Gamma_{\Omega}}=0
\end{array}\right.
$$

We set

$$
\mathscr{U}_{\text {ad }}=\{u(x) \in U=\{a, b\} \mid u(\cdot) \text { is measurable on } \Omega\}
$$

and

$$
J(u)=\int_{\Omega}\left\{(u(x)-\bar{u}(x))^{2}\left(y_{u}(x)-y_{d}(x)\right)^{2}+\left(u(x)-u_{d}(x)\right)^{2}\right\} d x,
$$

where $0<a<b$ and

$$
u_{d}(x)= \begin{cases}a+(1 / 4)(b-a), & x \in E_{1}, \\ a+(3 / 4)(b-a), & x \in E_{2} .\end{cases}
$$


We have that $E_{1} \cup E_{2}=\Omega, E_{1} \cap E_{2}=\emptyset, y_{d}(x)$ is a solution of Problem (3.2) corresponding to $u_{d}$ and

$$
\bar{u}(x)=a \chi_{E_{1}}(x)+b \chi_{E_{2}}(x) .
$$

It is clear that

$$
J(\bar{u}(\cdot))=\inf \left\{J(u(\cdot)) \mid u(\cdot) \in \mathscr{U}_{\mathrm{ad}}\right\}
$$

Let

$$
\mathscr{U}=\{u(x) \in[a, b] \mid u(\cdot) \text { is measurable on } \Omega\},
$$

then

$$
J\left(u_{d}(\cdot)\right)=\inf \{J(u(\cdot)) \mid u(\cdot) \in \mathscr{U}\} .
$$

We thus have $\bar{u}(x) \neq u_{d}(x)$.

This example indicates that the optimal control of Problem E is not equal to the optimal control of the convexification problem for Problem E. It therefore implies that we can't use the convexification method alone to solve both Problems $\mathrm{E}$ and $\mathrm{E}_{Q}$.

We shall now discuss Problem $\mathrm{E}_{Q}$. Suppose that

(P3) $Q$ is a closed and convex subset of $H_{0}^{1}(\Omega)$.

Let

$$
d_{Q}(y)=\min \left\{\|y-q\|_{H^{\prime}(\Omega)} \mid q \in Q\right\} \quad \text { and } \quad d_{U}(u(x))=\min _{v \in U}|v-u(x)|,
$$

where $|u|=\left\{\sum_{i=1}^{n} u_{i}^{2}\right\}^{1 / 2}$ and $u=\left\{u_{1}, \ldots, u_{n}\right\}$.

DEFINITION 3.1. Let $Z$ be a Banach space. A set $S$ is said to be finite codimensional in $Z$ if there exists a point $z \in S$ such that $Z_{0} \triangleq \operatorname{span}(S-z)$ is a finite codimensional subspace of $Z$ and $\overline{c o}(S-z)$ has a nonempty interior in $Z_{0}$.

We have the following result.

THEOREM 3.1. Let (P1)-(P3) hold, $\bar{u}(\cdot) \in \mathscr{U}_{a d}^{Q}$ be an optimal control of Problem $\mathrm{E}_{Q}$, $\bar{y}(\cdot)=y(\cdot, \bar{u})$ be an optimal state and $Q$ be finite codimensional in $H_{0}^{1}(\Omega)$. Then there exist $\psi^{0} \in[-1,0], \psi^{1} \in[-1,0], \xi(x)=\left(\xi^{1}(x), \ldots, \xi^{m}(x)\right), \varphi \in H^{-1}, \psi \in H_{0}^{1}(\Omega)$, such that

$$
\begin{gathered}
\left(\psi^{0}, \psi^{1}, \varphi\right) \neq 0, \\
\langle\varphi, q(\cdot)-\bar{y}(\cdot)\rangle \leq 0, \quad \forall q \in Q
\end{gathered}
$$


and $\psi(\cdot)$ satisfies the following equation

$$
\left\{\begin{aligned}
-\sum_{i, j=1}^{n} & \frac{\partial}{\partial x_{i}}\left(a_{i j}(x, \bar{u}(x)) \frac{\partial}{\partial x_{j}} \psi(x)\right) \\
= & f_{y}(x, \bar{y}(x), \bar{u}(x)) \psi(x)+\psi^{0}\left[f_{y}^{0}(x, \bar{y}(x), \nabla \bar{y}(x), \bar{u}(x))\right. \\
& \left.-\sum_{i=1}^{n} \frac{\partial}{\partial x_{i}} f_{\zeta_{i}}^{0}(x, \bar{y}(x), \nabla \bar{y}(x), \bar{u}(x))\right]-\varphi \\
\left.\psi\right|_{\Gamma_{\Omega}}= & 0
\end{aligned}\right.
$$

and the maximum condition

$$
K(x) \cdot \bar{u}(x)=\max _{v \in U} K(x) \cdot v, \quad \text { a.e. } \quad x \in \Omega
$$

holds, where

$$
\begin{gathered}
H(x, u)=-\sum_{i, j=1}^{n} a_{i j}(x, u) \frac{\partial}{\partial x_{i}} \bar{y}(x) \frac{\partial}{\partial x_{j}} \psi(x) \\
+\psi(x) f(x, \bar{y}(x), u)+\psi^{0} f^{0}(x, \bar{y}(x), \nabla \bar{y}(x), u), \\
N(x) \in \partial_{u} H(x, \bar{u}(x)) \text { and } \\
K(x)=N(x)+\psi^{1} \xi(x) .
\end{gathered}
$$

Here $\xi^{i}(x)=1$ as $\bar{u}^{i}(x)=a^{i}$ and $\xi^{i}(x)=-1$ as $\bar{u}^{i}(x)=b^{i}$, in which $\left[a^{i}, b^{i}\right]=$ $\operatorname{Proj}_{X_{i}} \operatorname{co} U, \bar{u}(x)=\left(\bar{u}^{1}(x), \ldots, \bar{u}^{m}(x)\right)$.

PROOF. Now, for any $u(\cdot), v(\cdot) \in \mathscr{U}$, we define $d(u(\cdot), v(\cdot))$ by

$$
d(u(\cdot), v(\cdot))=\underset{\Omega}{\operatorname{esssup}}|u(x)-v(x)| .
$$

We know then that $(\mathscr{U}, d)$ is a complete metric space. Without loss of generality, we may assume that $J(\bar{u})=0$. For any $\varepsilon>0$, we define $F_{\varepsilon}: \mathscr{U} \rightarrow R$ by

$$
F_{\varepsilon}(u(\cdot))=\left\{\left[(J(u(\cdot))+\varepsilon)^{+}\right]^{2}+\left[\int_{\Omega} d_{U}(u(x)) d x\right]^{2}+\left[d_{Q}(y(\cdot ; u))\right]^{2}\right\}^{1 / 2},
$$

so that $F_{\varepsilon}:(\mathscr{U}, d) \rightarrow R$ is continuous. Furthermore, we have

$$
\begin{gathered}
F_{\varepsilon}(u(\cdot))>0, \quad \forall u(\cdot) \in \mathscr{U}, \\
F_{\varepsilon}(\bar{u}(\cdot))=\varepsilon \leq \inf \left\{F_{\varepsilon}(u(\cdot)) \mid u(\cdot) \in \mathscr{U}\right\}+\varepsilon .
\end{gathered}
$$


Hence, by Ekeland's variational principle (see [6]), we can find $u_{\varepsilon}(\cdot) \in \mathscr{U}$, such that

$$
\left\{\begin{aligned}
d\left(\bar{u}(\cdot), u_{\varepsilon}(\cdot)\right) & \leq \sqrt{\varepsilon}, \quad F_{\varepsilon}\left(u_{\varepsilon}(\cdot)\right) \leq F_{\varepsilon}(\bar{u}(\cdot)), \\
-\sqrt{\varepsilon} d\left(u(\cdot), u_{\varepsilon}(\cdot)\right) & \leq F_{\varepsilon}(u(\cdot))-F_{\varepsilon}\left(u_{\varepsilon}(\cdot)\right), \quad \forall u(\cdot) \in \mathscr{U} .
\end{aligned}\right.
$$

Let $z_{\varepsilon}(\cdot) \in H_{0}^{1}(\Omega)$ satisfy the following relation

$$
\left\{\begin{aligned}
-\sum_{i, j=1}^{n} & \frac{\partial}{\partial x_{i}}\left(a_{i j}\left(x, u_{\varepsilon}(x)\right) \frac{\partial}{\partial x_{j}} z_{\varepsilon}(x)\right. \\
= & f_{y}\left(x, y_{\varepsilon}(x), u_{\varepsilon}(x)\right) z_{\varepsilon}(x) \\
& +\sum_{i, j=1}^{n} \frac{\partial}{\partial x_{i}}\left[\left(a_{i j}(x, u(x))-a_{i j}\left(x, u_{\varepsilon}(x)\right)\right) \frac{\partial}{\partial x_{j}} y_{\varepsilon}(x)\right] \\
& +f\left(x, y_{\varepsilon}(x), u(x)\right)-f\left(x, y_{\varepsilon}(x), u_{\varepsilon}(x)\right) \\
\left.z_{\varepsilon}\right|_{\Gamma_{\Omega}}= & 0
\end{aligned}\right.
$$

and let

$$
\begin{aligned}
z_{\varepsilon}^{0}= & \int_{\Omega}\left\{f_{y}^{0}\left(x, y_{\varepsilon}(x), \nabla y_{\varepsilon}(x), u_{\varepsilon}(x)\right) z_{\varepsilon}(x)\right. \\
& +\sum_{i=1}^{n} f_{\zeta_{i}}^{0}\left(x, y_{\varepsilon}(x), \nabla y_{\varepsilon}(x), u_{\varepsilon}(x)\right) \frac{\partial}{\partial x_{i}} z_{\varepsilon}(x) \\
& \left.+f^{0}\left(x, y_{\varepsilon}(x), \nabla y_{\varepsilon}(x), u(x)\right)-f^{0}\left(x, y_{\varepsilon}(x), \nabla y_{\varepsilon}(x), u_{\varepsilon}(x)\right)\right\} d x
\end{aligned}
$$

We set

$$
y_{u}(x)=y(x ; u) \quad \text { and } \quad y_{\varepsilon}(x)=y\left(x ; u_{\varepsilon}\right) .
$$

By Theorem 2.1, we have

$$
\left\{\begin{array}{l}
y_{u}(x)=y_{\varepsilon}(x)+z_{\varepsilon}(x)+r_{\varepsilon}(x), \\
J(u)=J\left(u_{\varepsilon}\right)+z_{\varepsilon}^{0}+r_{\varepsilon}^{0}
\end{array}\right.
$$

and

$$
\left\|r_{\varepsilon}\right\|_{H^{\prime}(\Omega)}=o\left(\left\|u-u_{\varepsilon}\right\|_{L^{\infty}(\Omega)}\right), \quad\left|r_{\varepsilon}^{0}\right|=o\left(\left\|u-u_{\varepsilon}\right\|_{L^{\infty}(\Omega)}\right) .
$$

From (3.10), we obtain that

$$
-\sqrt{\varepsilon} d\left(u, u_{\varepsilon}\right) \leq F_{\varepsilon}(u)-F_{\varepsilon}\left(u_{\varepsilon}\right)
$$




$$
\begin{aligned}
= & \frac{1}{B_{\varepsilon}}\left[(J(u)+\varepsilon)^{+}+\left(J\left(u_{\varepsilon}\right)+\varepsilon\right)^{+}\right]\left[(J(u)+\varepsilon)^{+}-\left(J\left(u_{\varepsilon}\right)+\varepsilon\right)^{+}\right] \\
& +\frac{1}{B_{\varepsilon}} \int_{\Omega}\left[d_{U}(u(x))+d_{U}\left(u_{\varepsilon}(x)\right)\right] d x \int_{\Omega}\left[d_{U}(u(x))-d_{U}\left(u_{\varepsilon}(x)\right)\right] d x \\
& +\frac{1}{B_{\varepsilon}}\left[d_{Q}(y(\cdot ; u))+d_{Q}\left(y\left(\cdot ; u_{\varepsilon}\right)\right)\right]\left[d_{Q}(y(\cdot ; u))-d_{Q}\left(y\left(\cdot ; u_{\varepsilon}\right)\right)\right],
\end{aligned}
$$

where $B_{\varepsilon}=F_{\varepsilon}(u)+F_{\varepsilon}\left(u_{\varepsilon}\right)$. We define

$$
\left\{\begin{array}{l}
\varphi_{\varepsilon}^{0}=\frac{1}{F_{\varepsilon}\left(u_{\varepsilon}\right)}\left(J\left(u_{\varepsilon}\right)+\varepsilon\right)^{+} \\
\varphi_{\varepsilon}^{1}=\frac{1}{F_{\varepsilon}\left(u_{\varepsilon}\right)} \int_{\Omega} d_{U}\left(u_{\varepsilon}(x)\right) d x \\
\varphi_{\varepsilon}=\frac{1}{F_{\varepsilon}\left(u_{\varepsilon}\right)} d_{Q}\left(y\left(\cdot ; u_{\varepsilon}\right)\right) \partial d_{Q}\left(y\left(\cdot ; u_{\varepsilon}\right)\right) \\
\xi_{\varepsilon}(x)=\left(\xi_{\varepsilon}^{1}(x), \ldots, \xi_{\varepsilon}^{m}(x)\right)
\end{array}\right.
$$

in which $\xi_{\varepsilon}^{i}(x)=1$ as $\bar{u}_{\varepsilon}^{i}(x) \in\left[a^{i}, a^{i}+\varepsilon\right]$ and $\xi_{\varepsilon}^{i}(x)=-1$ as $\bar{u}_{\varepsilon}^{i}(x) \in\left[b^{i}-\varepsilon, b^{i}\right]$, $\left[a^{i}, b^{i}\right]=\operatorname{Proj}_{X_{i}} \operatorname{co} U, \bar{u}_{\varepsilon}(x)=\left(\bar{u}_{\varepsilon}^{1}(x), \ldots, \bar{u}_{\varepsilon}^{m}(x)\right)$. From (3.14), we obtain that

$$
\begin{aligned}
-\sqrt{\varepsilon} d\left(u, u_{\varepsilon}\right) \leq & \varphi_{\varepsilon}^{0} z_{\varepsilon}^{0}+\varphi_{\varepsilon}^{1} \int_{\Omega} \xi_{\varepsilon}(x)\left[u(x)-u_{\varepsilon}(x)\right] d x \\
& +\left\langle\varphi_{\varepsilon}, y_{\varepsilon}\right\rangle_{H^{-1}, H^{\prime}}+o\left(\left\|u-u_{\varepsilon}\right\|_{L^{\infty}(\Omega)}\right)
\end{aligned}
$$

Let $\psi_{\varepsilon}^{0}=-\varphi_{\varepsilon}^{0}, \psi_{\varepsilon}^{1}=-\varphi_{\varepsilon}^{1}$ and

$$
\left\{\begin{aligned}
&-\sum_{i, j=1}^{n} \frac{\partial}{\partial x_{i}}\left(a_{i j}\left(x, u_{\varepsilon}(x)\right) \frac{\partial}{\partial x_{j}} \psi_{\varepsilon}(x)\right) \\
&= f_{y}\left(x, y_{\varepsilon}(x), u_{\varepsilon}(x)\right) \psi_{\varepsilon}(x)+\psi_{\varepsilon}^{0}\left[f_{y}^{0}\left(x, y_{\varepsilon}(x), \nabla y_{\varepsilon}(x), u_{\varepsilon}(x)\right)\right. \\
&\left.-\sum_{i=1}^{n} \frac{\partial}{\partial x_{i}} f_{\zeta_{i}}^{0}\left(x, y_{\varepsilon}(x), \nabla y_{\varepsilon}(x), u_{\varepsilon}(x)\right)\right]-\varphi_{\varepsilon} \\
&\left.\psi_{\varepsilon}\right|_{\Gamma_{\Omega}}=0
\end{aligned}\right.
$$

From (3.11), (3.12) and (3.17), we have

$$
\begin{aligned}
\varphi_{\varepsilon}^{0} z_{\varepsilon}^{0}= & -\psi_{\varepsilon}^{0} z_{\varepsilon}^{0} \\
= & -\int_{\Omega} \psi_{\varepsilon}^{0}\left[f_{y}^{0}\left(x, y_{\varepsilon}(x), \nabla y_{\varepsilon}(x), u_{\varepsilon}(x)\right)\right. \\
& \left.-\sum_{i=1}^{n} \frac{\partial}{\partial x_{i}} f_{\zeta_{i}}^{0}\left(x, y_{\varepsilon}(x), \nabla y_{\varepsilon}(x), u_{\varepsilon}(x)\right)\right] z_{\varepsilon}(x) d x
\end{aligned}
$$




$$
\begin{aligned}
& -\int_{\Omega} \psi_{\varepsilon}^{0}\left[f^{0}\left(x, y_{\varepsilon}(x), \nabla y_{\varepsilon}(x), u(x)\right)-f^{0}\left(x, y_{\varepsilon}(x), \nabla y_{\varepsilon}(x), u_{\varepsilon}(x)\right)\right] d x \\
= & -\int_{\Omega}\left\{\sum_{i, j=1}^{n} a_{i j}\left(x, u_{\varepsilon}(x)\right) \frac{\partial}{\partial x_{i}} z_{\varepsilon}(x) \frac{\partial}{\partial x_{j}} \psi_{\varepsilon}(x)\right. \\
& \left.-f_{y}\left(x, y_{\varepsilon}(x), u_{\varepsilon}(x)\right) \psi_{\varepsilon}(x) z_{\varepsilon}(x)\right\} d x-\left\langle\varphi_{\varepsilon}, z_{\varepsilon}\right\rangle \\
& -\int_{\Omega} \psi_{\varepsilon}^{0}\left[f^{0}\left(x, y_{\varepsilon}(x), \nabla y_{\varepsilon}(x), u(x)\right)-f^{0}\left(x, y_{\varepsilon}(x), \nabla y_{\varepsilon}(x), u_{\varepsilon}(x)\right)\right] d x \\
= & \int_{\Omega}\left\{\sum _ { i , j = 1 } ^ { n } \left[a_{i j}\left(x, u(x)-a_{i j}\left(x, u_{\varepsilon}(x)\right)\right] \frac{\partial}{\partial x_{i}} z_{\varepsilon}(x) \frac{\partial}{\partial x_{j}} \psi_{\varepsilon}(x)\right.\right. \\
& -\psi_{\varepsilon}(x)\left[f\left(x, y_{\varepsilon}(x), u\right)-f\left(x, y_{\varepsilon}(x), u_{\varepsilon}(x)\right)\right] \\
& \left.-\psi_{\varepsilon}^{0}\left[f^{0}\left(x, y_{\varepsilon}(x), \nabla y_{\varepsilon}(x), u(x)\right)-f^{0}\left(x, y_{\varepsilon}(x), \nabla y_{\varepsilon}(x), u_{\varepsilon}(x)\right)\right]\right\} d x \\
& -\left\langle\varphi_{\varepsilon}, z_{\varepsilon}\right\rangle \\
= & -\int_{\Omega}\left[H(x, u(x), \varepsilon)-H\left(x, u_{\varepsilon}(x), \varepsilon\right)\right] d x-\left\langle\varphi_{\varepsilon}, z_{\varepsilon}\right\rangle,
\end{aligned}
$$

where

$$
\begin{aligned}
H(x, u, \varepsilon)= & -\sum_{i, j=1}^{n} a_{i j}(x, u) \frac{\partial}{\partial x_{i}} y_{\varepsilon}(x) \frac{\partial}{\partial x_{j}} \psi_{\varepsilon}(x) \\
& +\psi_{\varepsilon}(x) f\left(x, y_{\varepsilon}(x), u\right)+\psi_{\varepsilon}^{0} f^{0}\left(x, y_{\varepsilon}(x), \nabla y_{\varepsilon}(x), u\right)
\end{aligned}
$$

Substituting (3.18) into (3.16), we obtain

$$
\begin{aligned}
-\sqrt{\varepsilon} d\left(u, u_{\varepsilon}\right) \leq & \int_{\Omega}\left\{-\left[H(x, u(x), \varepsilon)-H\left(x, u_{\varepsilon}(x), \varepsilon\right)\right]\right. \\
& \left.-\psi_{\varepsilon}^{1} \xi_{\varepsilon}(x)\left[u(x)-u_{\varepsilon}(x)\right]\right\} d x+o\left(\left\|u-u_{\varepsilon}\right\|_{L^{\infty}(\Omega)}\right) .
\end{aligned}
$$

It is clear that

$$
\left(\psi_{\varepsilon}^{0}\right)^{2}+\left(\psi_{\varepsilon}^{1}\right)^{2}+\left\|\varphi_{\varepsilon}\right\|_{H^{-1}}^{2}=1
$$

So there exist $\psi^{0} \in[-1,0], \psi^{1} \in[-1,0], \varphi_{\varepsilon} \in H^{-1}$ and a sequence of $\left\{\psi_{\varepsilon_{i}}^{0}, \psi_{\varepsilon_{i}}^{1}, \varphi_{\varepsilon_{i}}\right\}$, such that

$$
\left\{\psi_{\varepsilon_{i}}^{0}, \psi_{\varepsilon_{i}}^{1}, \varphi_{\varepsilon_{i}}\right\} \rightarrow\left\{\psi^{0}, \psi^{1} \varphi\right\} \quad \text { weakly star. }
$$

Since $d\left(u_{\varepsilon}(\cdot), \bar{u}(\cdot)\right) \leq \sqrt{\varepsilon}$, when $\varepsilon \rightarrow 0$, we have

$$
\left\|y_{\varepsilon}-\bar{y}\right\|_{H^{1}(\Omega)} \rightarrow 0
$$


It is known that there exists $\psi \in H_{0}^{1}(\Omega)$, which satisfies

$$
\left\{\begin{aligned}
-\sum_{i, j=1}^{n} & \frac{\partial}{\partial x_{i}}\left(a_{i j}(x, \bar{u}(x)) \frac{\partial}{\partial x_{j}} \psi(x)\right) \\
= & f_{y}(x, \bar{y}(x), \bar{u}(x)) \psi(x)+\psi^{0}\left[f_{y}^{0}(x, \bar{y}(x), \nabla \bar{y}(x), \bar{u}(x))\right. \\
& \left.-\sum_{i=1}^{n} \frac{\partial}{\partial x_{i}} f_{\zeta_{i}}^{0}(x, \bar{y}(x), \nabla \bar{y}(x), \bar{u}(x))\right]-\varphi \\
\left.\psi\right|_{\Gamma_{\Omega}} & =0
\end{aligned}\right.
$$

Let

$$
\left\{\begin{array}{l}
H(x, u)=H_{1}(x, u)+H_{2}(x, u), \\
H_{1}(x, u)=-\sum_{i, j=1}^{n} a_{i j}(x, u) \frac{\partial}{\partial x_{i}} \bar{y}(x) \frac{\partial}{\partial x_{j}} \psi(x), \\
H_{2}(x, u)=\psi(x) f(x, \bar{y}(x), u)+\psi^{0} f^{0}(x, \bar{y}(x), \nabla \bar{y}(x), u) .
\end{array}\right.
$$

From (3.19), we obtain

$$
\begin{aligned}
& -\sqrt{\varepsilon} d\left(u, u_{\varepsilon}\right)-\int_{\Omega}\left\{\left[H(x, u(x))-H\left(x, u_{\varepsilon}(x)\right]-\left[H(x, u(x), \varepsilon)-H\left(x, u_{\varepsilon}(x), \varepsilon\right)\right]\right\} d x\right. \\
& \quad \leq \int_{\Omega}\left\{-\left[H(x, u(x))-H\left(x, u_{\varepsilon}(x)\right)\right]-\psi_{\varepsilon}^{1} \xi_{\varepsilon}(x)\left[u(x)-u_{\varepsilon}(x)\right]\right\} d x \\
& \quad+o\left(\left\|u-u_{\varepsilon}\right\|_{L^{\infty}(\Omega)}\right) .
\end{aligned}
$$

We may therefore obtain

$$
\int_{\Omega}\left\{\left[H(x, u(x))-H\left(x, u_{\varepsilon}(x)\right]-\left[H(x, u(x), \varepsilon)-H\left(x, u_{\varepsilon}(x), \varepsilon\right)\right]\right\} d x \leq C \Delta_{\varepsilon} d\left(u, u_{\varepsilon}\right),\right.
$$

where

$$
\begin{aligned}
\Delta_{\varepsilon} \triangleq & \left\|\bar{y}-y_{\varepsilon}\right\|_{H^{1}(\Omega)}+\left|\psi^{0}-\psi_{\varepsilon}^{0}\right| \\
& +\left|\int_{\Omega} \sum_{i, j=1}^{n} a_{i j}(x, \bar{u}(x)) \frac{\partial}{\partial x_{i}} \bar{y}(x) \frac{\partial}{\partial x_{j}}\left(\psi(x)-\psi_{\varepsilon}(x)\right) d x\right| .
\end{aligned}
$$

It is easy to see that $\Delta_{\varepsilon} \rightarrow 0$ as $\varepsilon \rightarrow 0$. From (3.21), we have

$$
\begin{aligned}
-\left(\sqrt{\varepsilon}+C \Delta_{\varepsilon}\right) d\left(u, u_{\varepsilon}\right) \leq & \int_{\Omega}\left\{-\left[H(x, u(x))-H\left(x, u_{\varepsilon}(x)\right)\right]\right. \\
& \left.-\psi_{\varepsilon}^{1} \xi_{\varepsilon}(x)\left[u(x)-u_{\varepsilon}(x)\right]\right\} d x+o\left(\left\|u-u_{\varepsilon}\right\|_{L^{\infty}(\Omega)}\right) .
\end{aligned}
$$


For any $v \in \mathscr{U}$, let

$$
u(x)=u_{\varepsilon}(x)+\rho\left(v(x)-u_{\varepsilon}(x)\right),
$$

then $d\left(u, u_{\varepsilon}\right)=\rho d\left(v, u_{\varepsilon}\right)$. From (3.22), we have

$$
-\left(\sqrt{\varepsilon}+C \Delta_{\varepsilon}\right) d\left(v, u_{\varepsilon}\right) \leq \int_{\Omega}\left\{-N_{\varepsilon}(x)-\psi_{\varepsilon}^{1} \xi_{\varepsilon}(x)\right\}\left[v(x)-u_{\varepsilon}(x)\right] d x,
$$

where

$$
\begin{gathered}
N_{\varepsilon}(x)=\sum_{i, j=1}^{n} N_{\varepsilon}^{i j}(x) \frac{\partial}{\partial x_{i}} \bar{y}(x) \frac{\partial}{\partial x_{j}} \psi(x)+N_{2 \varepsilon}(x), \\
N_{\varepsilon}^{i j}(x) \in \partial_{u} a_{i j}\left(x, u_{\varepsilon}(x)\right) \quad \text { and } \quad N_{2 \varepsilon}(x) \in \partial_{u} H_{2}\left(x, u_{\varepsilon}(x)\right) .
\end{gathered}
$$

We shall now discuss the case as $\varepsilon \rightarrow 0$. We first want to prove that

$$
\left\{\psi^{0}, \psi^{1}, \varphi\right\} \neq 0
$$

In fact, if $\psi^{0} \neq 0$ or $\psi^{1} \neq 0$ then (3.24) holds. We shall suppose that $\psi^{0}=\psi^{1}=0$ and attempt to prove that $\varphi \neq 0$. In fact, according to the definition of the subdifferential, we have

$$
\left\langle\partial d_{Q}\left(y_{\varepsilon}(\cdot)\right), q(\cdot)-y_{\varepsilon}(\cdot)\right\rangle \leq 0, \quad \forall q(\cdot) \in Q,
$$

which implies that

$$
\left\langle\varphi_{\varepsilon}, q(\cdot)-y_{\varepsilon}(\cdot)\right\rangle \leq 0, \quad \forall q(\cdot) \in Q
$$

Furthermore, we have

$$
\left.\delta_{\varepsilon} \triangleq\left\langle\varphi_{\varepsilon}, \bar{y}-y_{\varepsilon}(\cdot)\right)\right\rangle \leq\left\langle\varphi_{\varepsilon}, \bar{y}-q(\cdot)\right\rangle, \quad \forall q \in Q .
$$

Since $d\left(u_{\varepsilon}(\cdot), \bar{u}(\cdot)\right) \leq \sqrt{\varepsilon}$, when $\varepsilon \rightarrow 0$, we have

$$
\left\|y_{\varepsilon}-\bar{y}\right\|_{H^{\prime}(\Omega)} \rightarrow 0 .
$$

Thus $\delta_{\varepsilon}^{1} \rightarrow 0$ as $\varepsilon \rightarrow 0$. Since the set $Q$ is finite codimensional in $H_{0}^{1}(\Omega)$, from $[7,12]$, we know that $\varphi \neq 0$, so (3.24) holds, that is, (3.3) holds. Furthermore, we obtain (3.4) from (3.25).

Set

$$
I_{i}(u)=\int_{\Omega} H_{i}(x, u(x)) d x, \quad i=1,2 .
$$


Then

$$
\left\|N_{\varepsilon}^{i j}\right\|_{L^{\infty}(\Omega)} \leq C, \quad\left\|N_{2 \varepsilon}\right\|_{L^{\infty}(\Omega)} \leq C,
$$

so there exist $N^{i j} \in L^{\infty}(\Omega), N_{2} \in L^{\infty}(\Omega)$, such that

$$
N_{\varepsilon}^{i j} \rightarrow N^{i j}, \quad N_{2 \varepsilon} \rightarrow N_{2} \quad \text { weakly star in } L^{\infty}(\Omega) .
$$

According to $\left\|u_{\varepsilon}-\bar{u}\right\|_{L^{\prime}(\Omega)} \rightarrow 0$ and from [6], we know that $N_{2} \in \partial I_{2}(\bar{u})$, that is, $N_{2}(x) \in \partial_{u} H_{2}(x, \bar{u}(x))$. Furthermore, we have $N^{i j}(x) \in \partial_{u} a_{i j}(x, \bar{u}(x))$. We note that $\xi_{\varepsilon}$ is independent of $\varepsilon$, so there exists $\xi(x)$ such that $\lim _{\varepsilon \rightarrow 0} \xi_{\varepsilon}(x)=\xi(x)$. Let

$$
N(x)=\sum_{i, j=1}^{n} N^{i j}(x) \frac{\partial}{\partial x_{i}} \bar{y}(x) \frac{\partial}{\partial x_{j}} \psi(x)+N_{2}(x) .
$$

From (3.23), we have

$$
0 \leq \int_{\Omega}\left\{-N(x)-\psi^{1} \xi(x)\right\}[v(x)-\bar{u}(x)] d x .
$$

We shall now set

$$
K(x)=N(x)+\psi^{1} \xi(x)
$$

Applying Fillipov's Lemma, we have

$$
K(x) \cdot \bar{u}(x)=\max _{v \in \operatorname{coU} U} K(x) \cdot v=\max _{v \in U} K(x) \cdot v, \quad \text { a.e. } x \in \Omega .
$$

Theorem 3.1 is thus proved.

REMARK 3.1. In Theorem 3.1, if $Q=H_{0}^{1}(\Omega)$, then $\varphi=0$; If $U=\operatorname{co} U$, then $\psi^{1}=0$.

REMARK 3.2. In Theorem 3.1, we suppose that $Q$ is finite codimensional in $H_{0}^{1}(\Omega)$. If $Q$ is not finite codimensional in $H_{0}^{1}(\Omega)$, then Theorem 3.1 may be trivial.

EXAMPLE 3.2. We consider the system

$$
\begin{aligned}
& \left\{\begin{array}{l}
-\operatorname{div}[(1+u(x)) \nabla y(x)]=u(x), \quad x \in \Omega, \\
\left.y_{u}\right|_{\Gamma_{\Omega}}=0,
\end{array}\right. \\
& \mathscr{U}_{\mathrm{ad}}=\{u(x) \in[-1,1] \mid u(\cdot) \text { is measurable on } \Omega\}
\end{aligned}
$$


and we give a functional

$$
J(u)=\int_{\Omega}\left\{y_{u}(x)+u(x)\right\} d x .
$$

Let $u_{0}(x)=0$. Then $y_{0}(\cdot)=0$ is the solution of (3.26) corresponding to $u=u_{0}$. We shall now suppose that $Q=\left\{y_{0}(\cdot)=0\right\}$. It is clear that $\bar{u}(x)=0$ is the optimal control and $\bar{y}(x)=0$ is the optimal state. Suppose Theorem 3.1 holds. Then there exists $\left(\psi^{0}, \psi(\cdot)\right) \neq 0$, where $\psi(\cdot)$ satisfies

$$
\begin{aligned}
& \left\{\begin{array}{l}
-\Delta \psi(x)=\psi^{0}+\varphi, \quad x \in \Omega, \\
\left.\psi\right|_{\Gamma_{\Omega}}=0,
\end{array}\right. \\
& H(x, u)=\psi(x) u+\psi^{0} u
\end{aligned}
$$

and

$$
K(x)=\psi(x)+\psi^{0}
$$

such that

$$
0=\max _{u \in[-1,1]}\left[\psi(x)+\psi^{0}\right] u .
$$

From (3.28), we obtain $\psi(x)=-\psi^{0}=$ constant. From (3.27), we have $\psi(x)=$ $-\psi^{0}=0$, which contradicts the assumption that $\left(\psi^{0}, \psi(\cdot)\right) \neq 0$.

Example 3.2 indicates that if $Q$ is not finite codimensional in $H_{0}^{1}(\Omega)$, then it may be that $K(x)=0$, that is, Theorem 3.1 may be trivial. Therefore, the condition that $Q$ is finite codimensional in $H_{0}^{1}(\Omega)$ is necessary.

We will now discuss the equivalent constraint problem. We define the equivalent constraint to mean that for any $u \in \mathscr{U}_{\mathrm{ad}}, \int_{\Omega} u_{i}(x) d x=1$. Let

$$
\mathscr{W}_{\mathrm{ad}}=\left\{u \in \mathscr{U}_{\mathrm{ad}} \mid \int_{\Omega} u_{i}(x) d x=1, i=1, \ldots, m\right\} .
$$

Problem $E_{1}$. Find $\bar{u} \in \mathscr{W}_{\text {ad }}$, such that

$$
J(\bar{u})=\inf \left\{J(u) \mid u \in \mathscr{W}_{\mathrm{ad}}\right\} .
$$

To solve Problem $E_{1}$, we introduce a new state equation

$$
y_{1}(u)=\int_{\Omega} u(x) d x \in R^{m}
$$


and thus obtain a new system

$$
\left\{\begin{array}{l}
-\sum_{i, j=1}^{n} \frac{\partial}{\partial x_{i}}\left(a_{i j}(x, u(x)) \frac{\partial}{\partial x_{j}} y(x)\right)=f(x, y(x), u(x)), \\
y_{1}(u)=\int_{\Omega} u(x) d x \in R^{m} \\
\left.y\right|_{\Gamma_{\Omega}}=0
\end{array}\right.
$$

which has a solution $\left(y, y_{1}\right) \in H_{0}^{1}(\Omega) \times R^{m}$ for any $u \in \mathscr{U}$. Let

$$
Q_{1}=H_{0}^{1}(\Omega) \times(1, \ldots, 1) .
$$

Problem $\mathrm{E}_{1}$ then becomes the problem of finding $\bar{u} \in \mathscr{U}_{\mathrm{ad}}$, such that $\left(y, y_{1}\right) \in Q_{1}$ and

$$
J(\bar{u})=\inf \left\{J(u) \mid u \in \mathscr{U}_{\mathrm{ad}}\right\} .
$$

It is clear that $Q_{1}$ is finite codimensional in $H_{0}^{1}(\Omega) \times R^{m}$. We have the following variation equation

$$
\left\{\begin{array}{l}
-\sum_{i, j=1}^{n} \frac{\partial}{\partial x_{i}}\left(a_{i j}(x, \bar{u}(x)) \frac{\partial}{\partial x_{j}} z(x)\right)=f_{y}(x, \bar{y}(x), \bar{u}(x)) z(x) \\
\quad+\sum_{i, j=1}^{n} \frac{\partial}{\partial x_{i}}\left[\left(a_{i j}(x, v(x))-a_{i j}(x, \bar{u}(x))\right) \frac{\partial}{\partial x_{j}} \bar{y}(x)\right] \\
\quad+[f(x, \bar{y}(x), v(x))-f(x, \bar{y}(x), \bar{u}(x))], \\
z_{1}=\int_{\Omega}(v(x)-\bar{u}(x)) d x, \\
\left.z\right|_{\Gamma_{\Omega}}=0
\end{array}\right.
$$

and the following conjugate equation

$$
\left\{\begin{array}{l}
-\sum_{i, j=1}^{n} \frac{\partial}{\partial x_{i}}\left(a_{i j}(x, \bar{u}(x)) \frac{\partial}{\partial x_{j}} \psi(x)\right) \\
\quad=f_{y}(x, \bar{y}(x), \bar{u}(x)) \psi(x)+\psi^{0}\left[f_{y}^{0}(x, \bar{y}(x), \nabla \bar{y}(x), \bar{u}(x))\right. \\
\left.\quad-\sum_{i=1}^{n} \frac{\partial}{\partial x_{i}} f_{\zeta_{i}}^{0}(x, \bar{y}(x), \nabla \bar{y}(x), \bar{u}(x))\right], \\
\psi_{1}=\mu, \\
\left.\psi\right|_{\Gamma_{\Omega}}=0,
\end{array}\right.
$$

where $\mu=\left(v_{1}, \ldots, v_{m}\right)$ for $v_{i} \in[-1,1], i=1, \ldots, m$. The Hamiltonian function is

$$
H(x, u)=-\sum_{i, j=1}^{n} a_{i j}(x, u) \frac{\partial}{\partial x_{i}} \bar{y}(x) \frac{\partial}{\partial x_{j}} \psi(x)
$$




$$
+\psi(x) f(x, \bar{y}(x), u)+\psi^{0} f^{0}(x, \bar{y}(x), \nabla \bar{y}(x), u)+\langle\mu, u\rangle .
$$

THEOREM 3.2. Let (P1)-(P2) hold, $\bar{u}(\cdot) \in \mathscr{W}_{\text {ad }}$ be an optimal control of Problem $\mathrm{E}_{1}$ and $\bar{y}(\cdot)=y(\cdot, \bar{u})$ be an optimal state. Then there exist $\psi^{i} \in[-1,0], i=0,1$, $\mu=\left(v_{1}, \cdots, v_{m}\right), v_{i} \in[-1,1], i=1, \ldots, m, \xi(x)$ given by Theorem 3.1 and $\psi \in H_{0}^{1}(\Omega)$ satisfying (3.5), such that

$$
\left(\psi^{0}\right)^{2}+\left(\psi^{1}\right)^{2}+\|\mu\|^{2}=1 .
$$

Let

$$
\begin{aligned}
H(x, u)= & -\sum_{i, j=1}^{n} a_{i j}(x, u) \frac{\partial}{\partial x_{i}} \bar{y}(x) \frac{\partial}{\partial x_{j}} \psi(x) \\
& +\psi(x) f(x, \bar{y}(x), u)+\psi^{0} f^{0}(x, \bar{y}(x), \nabla \bar{y}(x), u)+\langle\mu, u\rangle, \\
& N(x) \in \partial_{u} H(x, \bar{u}(x))
\end{aligned}
$$

and

$$
K(x)=N(x)+\psi^{1} \xi(x)
$$

We have that

$$
K(x) \cdot \bar{u}(x)=\max _{v \in U} K(x) \cdot v, \quad \text { a.e. } x \in \Omega .
$$

We shall now return to Problem D which was raised in Section 1 and shall describe the optimal domain using the result of Theorem 3.2.

Suppose $\Omega_{\infty} \in \Pi$ (given by (1.3)) is an optimal domain. This implies that the function

$$
\bar{u}(x)=a \chi_{\Omega_{\sharp}}(x)+b \chi_{\Omega_{g}}(x)
$$

is an optimal control of Problem C. Thus the optimal state $\bar{y}(x)$ satisfies:

$$
\left\{\begin{array}{l}
-\operatorname{div}(\bar{u}(x) \nabla \bar{y}(x))=f(x), \quad x \in G, \\
\bar{y} \mid \Gamma_{G}=0 .
\end{array}\right.
$$

From Theorem 3.2, we know that there exist $\psi^{i} \in[-1,0], i=0,1, v \in[-1,1]$, $\xi(x)=\chi_{\Omega_{\Perp}}(x)-\chi_{\Omega_{\mathcal{I}}}(x)$ and $\psi \in H_{0}^{1}(\Omega)$, such that

$$
\left\{\begin{array}{l}
\left(\psi^{0}\right)^{2}+\left(\psi^{1}\right)^{2}+(\nu)^{2}=1, \\
-\operatorname{div}(\bar{u}(x) \nabla \psi(x))=\psi^{0}\left[f_{y}^{0}(x, \bar{y}(x), \nabla \bar{y}(x))-\sum_{i=1}^{n} \frac{\partial}{\partial x_{i}} f_{\zeta_{i}}^{0}(x, \bar{y}(x), \nabla \bar{y}(x))\right], \\
\left.\psi\right|_{\Gamma_{G}}=0 .
\end{array}\right.
$$


Let $K(x)=-\nabla \bar{y}(x) \cdot \nabla \psi(x)+\psi^{1} \xi(x)+\nu$. Then

$$
\bar{u}(x)= \begin{cases}b, & \text { if } \quad K(x)>0, \\ a, & \text { if } \quad K(x)<0\end{cases}
$$

that is,

$$
\{x \in G \mid K(x)<0\} \subset \Omega_{\mathscr{A}}, \quad\{x \in G \mid K(x)>0\} \subset \Omega_{\mathscr{B}} .
$$

\section{The case of $U$ being a closed set}

In this section, we consider the case of $U$ being a general closed set. Let

$$
d_{U}(u)=\inf \left\{\|v-u\|_{L^{\infty}(\Omega)} \mid v(\cdot) \in \mathscr{U}_{\mathrm{ad}}\right\}
$$

THEOREM 4.1. Let (P1)-(P3) hold, $\bar{u}(\cdot) \in \mathscr{U}_{\text {ad }}^{Q}$ be an optimal control of Problem $E_{Q}$ and $\bar{y}(\cdot)=y(\cdot, \bar{u})$ be an optimal state. Let $Q$ be finite codimensional in $H_{0}^{1}(\Omega)$. Then there exist $\psi^{0} \in[-1,0], \varphi \in H^{-1}, \xi \in L^{\infty}(\Omega)^{*}$ and $\psi \in H_{0}^{1}(\Omega)$, such that

$$
\begin{gathered}
\left(\psi^{0}, \xi, \varphi\right) \neq 0, \\
\langle\varphi, q(\cdot)-\bar{y}(\cdot)\rangle \leq 0, \quad \forall q \in Q,
\end{gathered}
$$

$\psi(\cdot)$ satisfies (3.5) and the following variational inequality holds:

$$
0 \leq \int_{\Omega} N(x)(\bar{u}(x)-v(x)) d x+\langle\xi, \bar{u}-v\rangle, \quad \forall v \in \mathscr{U}_{\mathrm{ad}},
$$

where $N(x)$ is given by (3.7)-(3.8).

PROOF. This proof is similar to the proof of Theorem 3.1. Without loss of generality, we may assume that $J(\bar{u})=0$. For any $\varepsilon>0$, we define $F_{\varepsilon}: \mathscr{U} \rightarrow R$ by

$$
\left.F_{\varepsilon}(u(\cdot))=\left\{\left[(J(u(\cdot))+\varepsilon)^{+}\right]^{2}+\left[d_{U}(u)\right)\right]^{2}+\left[d_{Q}(y(\cdot ; u))\right]^{2}\right\}^{1 / 2} .
$$

By Ekeland's variational principle (see [6]), we can find $u_{\varepsilon}(\cdot) \in \mathscr{U}$, such that

$$
\left\{\begin{aligned}
d\left(\bar{u}(\cdot), u_{\varepsilon}(\cdot)\right) & \leq \sqrt{\varepsilon}, \quad F_{\varepsilon}\left(u_{\varepsilon}(\cdot)\right) \leq F_{\varepsilon}(\bar{u}(\cdot)), \\
-\sqrt{\varepsilon} d\left(u(\cdot), u_{\varepsilon}(\cdot)\right) & \leq F_{\varepsilon}(u(\cdot))-F_{\varepsilon}\left(u_{\varepsilon}(\cdot)\right), \quad \forall u(\cdot) \in \mathscr{U} .
\end{aligned}\right.
$$

Let $z_{\varepsilon}(\cdot) \in H_{0}^{1}(\Omega)$ satisfy (3.11) and let $z_{\varepsilon}^{0}$ be given by (3.12). Set

$$
y_{u}(x)=y(x ; u) \text { and } y_{\varepsilon}(x)=y\left(x ; u_{\varepsilon}\right) .
$$


By Theorem 2.1, we have

$$
\left\{\begin{array}{l}
y_{u}(x)=y_{\varepsilon}(x)+z_{\varepsilon}(x)+r_{\varepsilon}(x), \\
J(u)=J\left(u_{\varepsilon}\right)+z_{\varepsilon}^{0}+r_{\varepsilon}^{0}
\end{array}\right.
$$

and

$$
\left\|r_{\varepsilon}\right\|_{H^{\prime}(\Omega)}=o\left(\left\|u-u_{\varepsilon}\right\|_{L^{\infty}(\Omega)}\right), \quad\left|r_{\varepsilon}^{0}\right|=o\left(\left\|u-u_{\varepsilon}\right\|_{L^{\infty}(\Omega)}\right) .
$$

From (4.5), we obtain

$$
\begin{aligned}
-\sqrt{\varepsilon} d\left(u, u_{\varepsilon}\right) \leq & F_{\varepsilon}(u)-F_{\varepsilon}\left(u_{\varepsilon}\right) \\
= & \frac{1}{B_{\varepsilon}}\left[(J(u)+\varepsilon)^{+}+\left(J\left(u_{\varepsilon}\right)+\varepsilon\right)^{+}\right]\left[(J(u)+\varepsilon)^{+}-\left(J\left(u_{\varepsilon}\right)+\varepsilon\right)^{+}\right] \\
& +\frac{1}{B_{\varepsilon}}\left[d_{U}(u)+d_{U}\left(u_{\varepsilon}\right)\right]\left[d_{U}(u)-d_{U}\left(u_{\varepsilon}\right)\right] \\
& +\frac{1}{B_{\varepsilon}}\left[d_{Q}(y(\cdot ; u))+d_{Q}\left(y\left(\cdot ; u_{\varepsilon}\right)\right)\right]\left[d_{Q}(y(\cdot ; u))-d_{Q}\left(y\left(\cdot ; u_{\varepsilon}\right)\right)\right]
\end{aligned}
$$

where $B_{\varepsilon}=F_{\varepsilon}(u)+F_{\varepsilon}\left(u_{\varepsilon}\right)$. We define

$$
\left\{\begin{array}{l}
\varphi_{\varepsilon}^{0}=\frac{1}{F_{\varepsilon}\left(u_{\varepsilon}\right)}\left(J\left(u_{\varepsilon}\right)+\varepsilon\right)^{+} \\
\xi_{\varepsilon}=\frac{1}{F_{\varepsilon}\left(u_{\varepsilon}\right)} d_{U}\left(u_{\varepsilon}\right) \partial d_{U}\left(u_{\varepsilon}\right) \\
\varphi_{\varepsilon}=\frac{1}{F_{\varepsilon}\left(u_{\varepsilon}\right)} d_{Q}\left(y\left(\cdot ; u_{\varepsilon}\right)\right) \partial d_{Q}\left(y\left(\cdot ; u_{\varepsilon}\right)\right) .
\end{array}\right.
$$

It is clear that

$$
\left(\psi_{\varepsilon}^{0}\right)^{2}+\left(\left\|\xi_{\varepsilon}\right\|_{\left(L^{\infty}(\Omega)\right)^{*}}\right)^{2}+\left(\left\|\varphi_{\varepsilon}\right\|_{H^{-1}}\right)^{2}=1 .
$$

From (4.8), we obtain that

$$
-\sqrt{\varepsilon} d\left(u, u_{\varepsilon}\right) \leq \varphi_{\varepsilon}^{0} z_{\varepsilon}^{0}+\left\langle\xi_{\varepsilon}, u-u_{\varepsilon}\right\rangle+o\left(\left\|u-u_{\varepsilon}\right\|_{L^{\infty}(\Omega)}\right) .
$$

Let $\psi_{\varepsilon}^{0}=-\varphi_{\varepsilon}^{0}$ and suppose that $\psi_{\varepsilon}(x)$ satisfies (3.17). Then from (3.11), (3.12) and (3.17), we have that

$$
\varphi_{\varepsilon}^{0} z_{\varepsilon}^{0}=-\int_{\Omega}\left[H(x, u(x), \varepsilon)-H\left(x, u_{\varepsilon}(x), \varepsilon\right)\right] d x,
$$

where

$$
H(x, u, \varepsilon)=-\sum_{i, j=1}^{n} a_{i j}(x, u) \frac{\partial}{\partial x_{i}} y_{\varepsilon}(x) \frac{\partial}{\partial x_{j}} \psi_{\varepsilon}(x)
$$




$$
+\psi_{\varepsilon}(x) f\left(x, y_{\varepsilon}(x), u\right)+\psi_{\varepsilon}^{0} f^{0}\left(x, y_{\varepsilon}(x), \nabla y_{\varepsilon}(x), u\right)
$$

Substituting (4.11) into (4.10), we obtain

$$
\begin{aligned}
-\sqrt{\varepsilon} d\left(u, u_{\varepsilon}\right) \leq & -\int_{\Omega}\left[H(x, u(x), \varepsilon)-H\left(x, u_{\varepsilon}(x), \varepsilon\right)\right] d x \\
& +\left\langle\xi_{\varepsilon}, u-u_{\varepsilon}\right\rangle+o\left(\left\|u-u_{\varepsilon}\right\|_{L^{\infty}(\Omega)}\right)
\end{aligned}
$$

In a manner similar to that used for Theorem 3.1 we have

$$
\begin{aligned}
-\left(\sqrt{\varepsilon}+C \Delta_{\varepsilon}\right) d\left(u, u_{\varepsilon}\right) \leq & -\int_{\Omega}\left[H(x, u(x))-H\left(x, u_{\varepsilon}(x)\right)\right] d x \\
& +\left\langle\xi_{\varepsilon}, u-u_{\varepsilon}\right\rangle+o\left(\left\|u-u_{\varepsilon}\right\|_{L^{\infty}(\Omega)}\right)
\end{aligned}
$$

For any $v \in \mathscr{U}$, let

$$
u(x)=u_{\varepsilon}(x)+\rho\left(v(x)-u_{\varepsilon}(x)\right),
$$

then $d\left(u, u_{\varepsilon}\right)=\rho d\left(v, u_{\varepsilon}\right)$. From (4.13), we have

$$
-\left(\sqrt{\varepsilon}+C \Delta_{\varepsilon}\right) d\left(v, u_{\varepsilon}\right) \leq \int_{\Omega}\left(-N_{\varepsilon}(x)\right)\left[v(x)-u_{\varepsilon}(x)\right] d x+\left\langle\xi_{\varepsilon}, v-u_{\varepsilon}\right\rangle
$$

where $N_{\varepsilon}(x) \in \partial_{u} H\left(x, u_{\varepsilon}(x)\right)$. In a manner similar to that used for Theorem 3.1, there exist $\psi^{0} \in[-1,0], \xi \in\left(L^{\infty}(\Omega)\right)^{*}, \varphi \in H^{-1}$, such that $\psi_{\varepsilon}^{0} \rightarrow \psi^{0}$ and

$$
\left\{\xi_{\varepsilon}, \varphi_{\varepsilon}\right\} \rightarrow\{\xi, \varphi\} \quad \text { weakly star, }
$$

such that (4.1) and (4.2) hold. From the proof of Theorem 3.1, we know that there exists $N(x) \in \partial_{u} H(x, \bar{u}(x))$ such that

$$
0 \leq \int_{\Omega} N(x)[\bar{u}(x)-v(x)] d x+\langle\xi, \bar{u}-v\rangle .
$$

Theorem 4.1 is thus proved.

\section{Acknowledgement}

This work was supported by the NSF of China, the Chinese State Education Commission Science Foundation. We also thank the referee whose helpful suggestions were greatly appreciated.

\section{References}

[1] N. U. Ahmed and K. L. Teo, Optimal Control of Distributed Parameter System (North Holland, New York, 1981). 
[2] J. F. Bonnans and E. Casas, "A boundary Pontryagin's principle for the optimal control of state constrained elliptic systems", Int. Ser. Numer. Math. 107 (1992) 241-249.

[3] J. F. Bonnans and D. Tiba, "Pontryagin principle in the control of semilinear elliptic variational inequalities", Appl. Math. Optim. 23 (1991) 299-312.

[4] E. Casas, "Optimal control in coefficients of elliptic equations with state constraints", Appl. Math. Optim. 26 (1992) 21-37.

[5] E. Casas and J. Yong, "Maximum principle for state-constrained optimal control problems governed by quasilinear elliptic equation", Diff. Int. Eqn. 8 (1995) 1-18.

[6] F. H. Clarke, Optimization and Nonsmooth Analysis (Wiley, New York, 1983).

[7] H. O. Fattorini and H. Frankowska, "Necessary conditions for infinite dimensional control problems", Math. Control Signal Systems 4 (1991) 41-67.

[8] D. Gilbarg and N. S. Trudinger, Elliptic Partial Differential Equations of Second Order, 2nd ed. (Springer, Berlin, 1983).

[9] O. A. Ladyzhenskaya and N. N. Ural'tseva, Linear and Quasilinear Elliptic Equations (Academic Press, New York, 1968).

[10] X. Li, "Vector-valued measure and the necessary conditions for the optimal control problems of linear systems", in Proceedings of IFAC 3rd Symposium on Control of Distributed Parameter Systems, (Toulouse, 1982).

[11] X. Li and J. Yong, "Necessary conditions of optimal control for distributed parameter systems", SIAM J. Control Optim. 29 (1991) 895-908.

[12] X. Li and J. Yong, Optimal Control Theory for Infinite Dimensional Systems (Birkhauser, Boston, 1995).

[13] J. L. Lions, Optimal Control of Systems Governed by Partial Differential Equations (Springer, New York, 1971).

[14] J. Yong, "Existence theory for optimal control of distributed parameter systems", Kodai Math. J. 15 (1992) 193-220.

[15] J. Yong, "Pontryagin maximum principle for semilinear second order elliptic partial differential equations and variational inequalities with state constraints", Diff. Int. Eqn. 5 (1992) 1307-1334.

[16] J. Yong, "Necessary conditions for minimax control problems of second order elliptic partial differential equations", Kodai Math. J. 16 (1993) 469-486. 